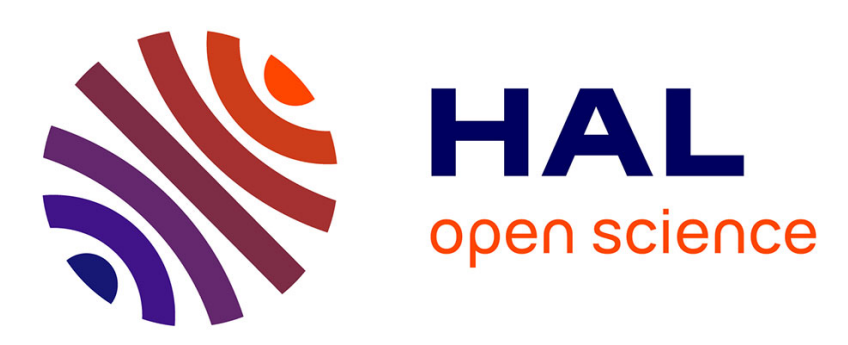

\title{
Dynamical systems complexity with a view towards air traffic management applications
}

Stéphane Puechmorel, Daniel Delahaye

\section{To cite this version:}

Stéphane Puechmorel, Daniel Delahaye. Dynamical systems complexity with a view towards air traffic management applications. CDC/CCC 2009, Joint 48th IEEE Conference on Decision and Control and 28th Chinese Control Conference, Dec 2009, Shanghai, China. pp 8369-8374, 10.1109/CDC.2009.5400549 . hal-01018355

\section{HAL Id: hal-01018355 \\ https://hal-enac.archives-ouvertes.fr/hal-01018355}

Submitted on 7 Jul 2014

HAL is a multi-disciplinary open access archive for the deposit and dissemination of scientific research documents, whether they are published or not. The documents may come from teaching and research institutions in France or abroad, or from public or private research centers.
L'archive ouverte pluridisciplinaire HAL, est destinée au dépôt et à la diffusion de documents scientifiques de niveau recherche, publiés ou non, émanant des établissements d'enseignement et de recherche français ou étrangers, des laboratoires publics ou privés. 


\section{Dynamical Systems Complexity with a view towards air traffic management applications}

\author{
Stephane Puechmorel \\ Applied Mathematics Laboratory \\ ENAC \\ 7, Avenue Edouard Belin \\ 31055 Toulouse, France \\ Email: puechmor@recherche.enac.fr
}

\author{
Daniel Delahaye \\ Applied Mathematics Laboratory \\ ENAC \\ 7, Avenue Edouard Belin \\ 31055 Toulouse, France \\ Email: delahaye@ recherche.enac.fr
}

\begin{abstract}
The growth of air traffic in future years requires a paradigm shift in the way the aircraft are controlled. Major innovative projects (SESAR in Europe, NexGen in USA) have started in order to define and implement control tools based on time-space constraints on aircraft trajectories. As a consequence, an increasing level of automation is expected. In this framework, it is of primary importance to be able to quantify the hardness to produce conflict free trajectories for a given situation and the robustness of the solution found. In this paper, a characterisation based on the lyapunov exponents of a dynamical system interpolating the observed data will be presented. A first part will be devoted to vector field fitting, and a second one to efficient lyapunov exponents computation. Then, some practical implementation issues will be discussed.
\end{abstract}

\section{INTRODUCTION}

The Air Traffic Management (ATM) system has to cope with an increasing number of flights, pushing the capacity to its limits. As an example, the average daily traffic above Europe was 26286 flights/day, with a peak traffic demand in excess of 31000 flights [1]. Although delays are kept low, it is expected from the same reference that capacity has to be extended in the future. Basically, two strategies can be devised : adapt the demand to capacity (slot-route allocation, collaborative decision making, ...) or adapt the capacity to the demand (Airspace design, 4D trajectory planning, autonomous aircraft, ...). The first approach can be used in the context of current ATM system, while innovative future designs will mainly follow the second strategy.

Currently, complexity of the traffic is measured only as an operational capacity : the maximum number of aircraft that controllers are willing to accept is fixed on a persector basis and complexity is assessed by comparing the real number of aircraft with the sector capacity. It must be noted that under some circumstances controllers will accept aircraft beyond the capacity threshold while rejecting traffic at other times although the number of aircraft is well below the maximum capacity. This simple fact clearly show that capacity as a crude complexity metric is not enough by itself to fully account for the controller's workload. In order to better quantify the complexity, geometric features of the traffic have to be included. Some works have been conducted on the subject, mainly with the idea of finding an indicator that measures the workload of human controller [2], [3].
These studies cleary show that traffic structure is one of the most influencial factor for controllers workload. Within the framework of future ATM systems, where a higher level of automation will be introduced and where autonomous or nearly autonomous aircraft will be present in the traffic, such indicators are no longer relevant. To adress this problem, it is needed to have a metric of complexity that is sensitive to the structure of the set of aircraft trajectories, but makes no assumption on how the traffic will be controlled. Such a structural measure is quite difficult to design, mainly because there is no agreement on what an intrinsic complexity can be. The new approach presented here is based on a dynamical system model of the traffic, namely we assume that the observed velocities and positions of aircraft are samples of an underlying smooth vector field. The complexity is then obtained by computing geometrical features of this field. Currently the Lyapunov exponents are used to produce a map of complexity, that is to give a value describing the level of sensitivity to perturbations at points sampled in the airspace.

It has to be noted that the methodology described here can be extended to a wider context than Air Traffic Management. As an example, a priori analysis of situations involving several autonomous or coordinated robots can be done using complexity, with the purpose of planning robust trajectories.

\section{THE VECTOR FIELD FITTING PROBLEM}

The first step towards air traffic complexity evaluation is to fit a vector field to the observed velocities at aircraft positions and at given sample times.

Assuming that $N$ observations have been made, the dataset is a finite sequence of $N$ triples $\left(t_{i}, x_{i}, v_{i}\right)_{i=1}^{N}$ with first component the sampling time, second component the observed position and third component the observed velocity. The interpolating problem is to find a smooth enough mapping $X: \mathbb{R} \times \mathbb{R}^{3} \rightarrow \mathbb{R}^{3}$ such that $\forall i=1 \ldots N, X\left(t_{i}, x_{i}\right)=v_{i}$. It is sometimes more convenient to solve instead a smoothing problem that is formulated as finding a mapping $X$ minimizing the error :

$$
E(X)=\sum_{i=1}^{N}\left\|v_{i}-X\left(t_{i}, x_{i}\right)\right\|^{2}
$$


Both the interpolating and the smoothing problems are illposed since it is clear that an infinite number of vector fields $X$ will satisfy the constraints. In order to obtain a unique solution, some assumptions have to be made on $X$. Basically, there is two ways to add more constraints :

- Add a criterion based on smoothness of $X$ and select the $X$ that realizes the minimum of the criterion while satisfying the interpolating/smoothing conditions.

- Restrict the possible $X$ to a family parametrized with a finite number of parameters.

\section{A. Time-Space spline interpolation}

In spline interpolation (or smoothing), we require that the vector field $X$ is optimal with respect to a smoothness criterion. Keeping in mind that our final goal is to fit a vector field to air traffic samples, the smoothness criterion can be build from operational considerations :

- From a spatial point of view, given a velocity $v$ at point $x$, it is expected that the velocity will not be very different from $v$ in a neighborood of $x$. Furthermore, since real traffic is supposed to be conflict free, at least in the short term, we may assume that aircraft tend to adjust their own velocities as beeing as close as possible to the mean velocity of their neighboors. This requirement has to be mitigated in an operational context as pointed out by one reviewer of this work since high discrepancy may exist if aircraft are separated in flight levels. This is somewhat taken into account by expressing aircraft velocities and position in separation norms instead of isotropic coordinates. It is well known that smooth vector fields satisfying this mean value principle are harmonic ones, that is such that $\Delta X=0$, with $\Delta$ the laplacian, acting only on spatial coordinates. It is then natural to seek for an optimal vector field $X$ that is "as harmonic as possible", or more formally minimizing :

$$
\int_{\mathbb{R}} \int_{\mathbb{R}^{3}}\|\Delta X(t, x)\|^{2} d x d t
$$

- From a temporal point of view, it is expected that the interpolating $X$ has slow variation (namely, aircraft acceleration is kept as low as possible), that is realizes the minimum of :

$$
\int_{\mathbb{R}} \int_{\mathbb{R}^{3}}\left\|\frac{\partial X}{\partial t}(t, x)\right\|^{2} d x d t
$$

Gathering the two terms, we obtain a final criterion

$$
E(X)=\int_{\mathbb{R}} \int_{\mathbb{R}^{3}}\left\|\frac{\partial X}{\partial t}(t, x)\right\|^{2}+\mu\|\Delta X(t, x)\|^{2} d x d t
$$

with $\mu$ a positive real number tuning the relative importance of harmonicity. The interpolating problem becomes in this context a minimization problem with equality constraints, namely find $X$ such that $E(X)$ is minimum and $X\left(t_{i}, x_{i}\right)=$ $v_{i}, i=1 \ldots N$. The smoothing problem is more or less similar, but is unconstrained, with criterion :

$$
E(X)+\sum_{i=1}^{N} \alpha_{i}\left\|X\left(t_{i}, x_{i}\right)-v_{i}\right\|^{2}
$$

where the $\alpha_{i}$ are weighting factors. This kind of problem falls in the general category of interpolating or smoothing $L$-splines. Before deriving a closed-form solution, we will recall some definitions.

Definition 1: A time-dependent measurable vector field is said to be square summable if :

$$
\int_{\mathbb{R}} \int_{\mathbb{R}^{3}}\|X(t, x)\|^{2} d x d t<+\infty
$$

The space of all square summable fields will be denoted as $L^{2} . L^{2}$ is an hilbert space with respect to the inner product :

$$
\langle X, Y\rangle_{L^{2}}=\int_{\mathbb{R}} \int_{\mathbb{R}^{3}}\langle X(t, x), Y(x, t)\rangle d x d t
$$

Definition 2: A vector field $X$ is said to admit a weak partial derivative with respect to coordinate $k$ if it exists a vector field denoted by $D_{k} X$ satisfying :

$$
\forall \phi \in \mathscr{S} \int_{\mathbb{R}} \int_{\mathbb{R}^{3}}\left\langle X, \frac{\partial}{\partial x_{k}} \phi\right\rangle d x d t=-\int_{\mathbb{R}} \int_{\mathbb{R}^{3}}\left\langle D_{k} X, \phi\right\rangle d x d t
$$

where $\mathscr{S}$ is the space of smooth and rapidly decreasing functions.

Definition 3: $\mathscr{W}$ is the vector space of fields $X$ such that :

$$
\int_{\mathbb{R}} \int_{\mathbb{R}^{3}}\left\|\frac{\partial X}{\partial t}(t, x)\right\|^{2}+\mu\|\Delta X(t, x)\|^{2} d x d t<+\infty
$$

where all derivatives are taken in a weak sense. $\mathscr{W}$ has a natural inner product :

$$
\langle X, Y\rangle_{\mathscr{W}}=\int_{\mathbb{R}} \int_{\mathbb{R}^{3}}\left\langle\frac{\partial X}{\partial t}, \frac{\partial Y}{\partial t}\right\rangle+\mu\langle\Delta X, \Delta Y\rangle d x d t
$$

but is not an Hilbert space since the inner product is not positive definite. However, we may quotient out by the vector subspace of $\mathscr{W}$ whose elements $X$ are such that :

$$
\frac{\partial X}{\partial t}=0 \quad \Delta X=0
$$

The resulting quotient space provided with the induced inner product becomes an Hilbert space, denoted by $W$.

Definition 4: A mapping $G: \mathbb{R} \times \mathbb{R}^{3} \rightarrow \mathbb{R}^{3} \otimes W$ is a vector reproducing kernel for $W$ if for any $X \in W$ and any $(t, x) \in$ $\mathbb{R} \times \mathbb{R}^{3}$ :

$$
\forall u \in \mathbb{R}^{3},\langle X(t, x), u\rangle=\langle G(t, x) u, X\rangle_{W}
$$

A vector reproducing kernel has to satisfy a symmetry property :

Proposition 1: For any $\left(t_{0}, x_{0}\right),\left(t_{1}, x_{1}\right), G\left(t_{0}, x_{0}, t_{1}, x_{1}\right)=$ $G\left(t_{1}, x_{1}, t_{0}, x_{0}\right)^{h}$ where $G\left(t_{1}, x_{1}, t_{0}, x_{0}\right)^{h}$ is the adjoint of $G\left(t_{1}, x_{1}, t_{0}, x_{0}\right)$.

Proof: Let $u, v$ be vectors. We have by the reproducing property :

$$
\begin{aligned}
& \left\langle G\left(t_{0}, x_{0}, t_{1}, x_{1}\right) u, v\right\rangle=\left\langle G\left(t_{1}, x_{1}\right) v, G\left(t_{0}, x_{0}\right) u\right\rangle_{W} \\
& =\left\langle G\left(t_{0}, x_{0}\right) u, G\left(t_{1}, x_{1}\right) v\right\rangle_{W}=\left\langle G\left(t_{1}, x_{1}, t_{0}, x_{0}\right) v, u\right\rangle \\
& =\left\langle G\left(t_{1}, x_{1}, t_{0}, x_{0}\right)^{h} u, v\right\rangle
\end{aligned}
$$

Let $G$ be a reproducing kernel for $W$. A constraint $X\left(t_{i}, x_{i}\right)=v_{i}$ can be rewritten as $\forall u \in \mathbb{R}^{3},\left\langle G\left(t_{i}, x_{i}\right) u, X\right\rangle_{W}=$ $v_{i}$ or equivalently, by taking a basis $\left(e_{1}, e_{2}, e_{3}\right)$ of 
$\mathbb{R}^{3}:\left\langle G\left(t_{i}, x_{i}\right) e_{k}, X\right\rangle_{W}=v_{i, k}, k=1,2,3$ with $v_{i, k}$ the $k$ th component of vector $v_{i}$. An elementary constraint $\left\langle G\left(t_{i}, x_{i}\right) e_{k}, X\right\rangle_{W}=v_{i, k}$ is satisfied on a closed affine space of codimension one, so that the complete constraint $X\left(t_{i}, x_{i}\right)=v_{i}$ is satisfied by a closed affine space of finite codimension. Since there is a finite number of constraints, the set of all vectors in $W$ satisfying all the constraints is again a closed affine space $Q$ of finite codimension. The solution of the interpolation problem is then easily obtained : since the orthogonal projection on a closed convex set in an Hilbert space is well defined, the field $X$ in $W$ with minimum norm and satisfying the constraints is the orthogonal projection of the zero vector on the affine space $Q$. We have then proved the proposition :

Proposition 2: If $W$ admits a vector reproducing kernel $G$, the solution of the interpolation problem is a linear combination :

$$
X=\sum_{i=1}^{N} G\left(t_{i}, x_{i}\right) \lambda_{i}
$$

with coefficients $\lambda_{i}$ being vectors from $\mathbb{R}^{3}$.

Some care must be taken when expressing $X: W$ is a quotient space so the solution to the original interpolation problem will be :

$$
X=\sum_{i=1}^{N} G\left(t_{i}, x_{i}\right) \lambda_{i}+K
$$

with $K$ satisfying (II.2) Instead of trying to find directly a vector reproducing kernel in $W$, we will seek for the solution of an easier problem, that is find a $G$ such that for all $\left(t_{0}, x_{0}\right)$ and all $u \in \mathbb{R}^{3}$ :

$$
\forall \phi \in \mathscr{S},\left\langle G\left(t_{0}, x_{0}\right) u, \phi\right\rangle_{W}=\left\langle\phi\left(t_{0}, x_{0}\right), u\right\rangle
$$

we assume high enough smoothness on $G$ (defering thus checking at the end of the computation), we may perform an integration by parts to obtain :

$$
\begin{aligned}
& \left\langle G\left(t_{0}, x_{0}\right) u, \phi\right\rangle_{W}= \\
& \int_{\mathbb{R}} \int_{\mathbb{R}^{3}}\left\langle\left(-\frac{\partial^{2}}{\partial t^{2}}+\Delta^{2}\right) G\left(t_{0}, x_{0}\right)(t, x) u, \phi(t, x)\right\rangle d t d x
\end{aligned}
$$

The first important consequence of the previous expression is that the vector kernel will take its value in the subspace of diagonal $3 \times 3$ matrices. It is thus enough to find a scalar valued function $\phi\left(t_{0}, x_{0}, t, x\right)$ such that :

$$
\begin{aligned}
& G\left(t_{0}, x_{0}, t, x\right)= \\
& \left(\begin{array}{ccc}
\phi\left(t_{0}, x_{0}, t, x\right) & 0 & 0 \\
0 & \phi\left(t_{0}, x_{0}, t, x\right) & 0 \\
0 & 0 & \phi\left(t_{0}, x_{0}, t, x\right)
\end{array}\right)
\end{aligned}
$$

The repoducing kernel condition in this new context appears then to be the requirement that in a distributional sense, $\phi$ is an elementary solution $\left(\right.$ at $\left.\left(t_{0}, x_{0}\right)\right)$ for the differential operator :

$$
L=-\frac{\partial^{2}}{\partial t^{2}}+\Delta^{2}
$$

that is :

$$
L \phi\left(t_{0}, x_{0}, t, x\right)=\delta_{t_{0}, x_{0}}
$$

Taking the Fourier transform with respect to $x$ only in $\mathscr{S}^{\prime}$ of both sides of the equation yields :

$$
-\frac{\partial^{2}}{\partial t^{2}} \phi\left(\widehat{t_{0}, x_{0}, t}, \xi\right)+\|\xi\|^{4} \phi\left(\widehat{t_{0}, x_{0}, t}, \xi\right)=\delta_{t_{0}} 1(\xi)
$$

where $\widehat{\phi}$ is the Fourier transform of $\phi$ with respect to $x$, $\xi$ is the Fourier variable and $1(\xi)$ is the constant function equal to 1 . A standard trick for finding $\widehat{\phi}$ is to introduce a jump at $t_{0}$ in the function or one of its derivative to make the $\delta_{t_{0}}$ appear. Here, the jump has to be in the first derivative, so that the solution is :

$$
\mathbf{p f}\left(\frac{1}{\sqrt{\mu}\|\xi\|^{2}}\right) \exp \left(-\left|t-t_{0}\right| \sqrt{\mu}|\xi|^{2}\right)
$$

The inverse Fourier transform of this distribution can be obtained readily by integration and has the form of a translate of a mother function :

$$
\phi\left(t_{0}, x_{0}, t, x\right)=p\left(t-t_{0}, x-x_{0}\right)
$$

The mother function $p$ is given by :

$$
\begin{aligned}
& p(t, x)=\frac{1}{8 \pi^{3}} \int_{\mathbb{R}^{3}} \frac{1}{\sqrt{\mu}\|\xi\|^{2}} \\
& \exp \left(-|t| \sqrt{\mu}\|\xi\|^{2}\right) \exp (i\langle x, \xi\rangle) d \xi
\end{aligned}
$$

by Fubini's theorem and polar change of variables it becomes :

$$
\begin{gathered}
p(t, x)=\frac{1}{8 \pi^{3} \sqrt{\mu}} \int_{\mathbb{R}} \exp \left(-|t| \sqrt{\mu} r^{2}\right) \\
\int_{\mathscr{S}^{2}} \exp (i\langle x, r s\rangle) d \sigma(s) d r
\end{gathered}
$$

with $d \sigma$ the solid angle measure. Using a polar parametrization of the unit sphere :

$$
\begin{aligned}
& p(t, x)= \\
& \frac{1}{8 \pi^{3} \sqrt{\mu}} \int_{\mathbb{R}} \exp \left(-|t| \sqrt{\mu} r^{2}\right) \\
& \int_{0}^{2 \pi} \int_{0}^{\pi} \exp (i\|x\| r \cos \theta) \sin \theta d \theta d \phi d r
\end{aligned}
$$

and finally :

$$
\begin{aligned}
& p(t, x)=\frac{1}{2 \pi^{2} \sqrt{\mu}} \\
& \int_{\mathbb{R}} \exp \left(-|t| \sqrt{\mu} r^{2}\right) \frac{\sin \|x\| r}{\|x\| r} d r
\end{aligned}
$$

by Parseval equality :

$$
\begin{aligned}
& p(t, x)=\frac{1}{4\|x\| \pi^{2}} \sqrt{\frac{\pi}{|t| \sqrt{\mu}}} \\
& \int_{-\frac{\|x\|}{2 \pi}}^{\frac{\|x\|}{2 \pi}} \exp \left(-\frac{\pi^{2} \omega^{2}}{|t| \sqrt{\mu}}\right) d \omega
\end{aligned}
$$

so in terms of error function erf :

$$
p(t, x)=\frac{1}{4 \pi^{2}\|x\|} \operatorname{erf}\left(\frac{\|x\|}{2 \sqrt{|t| \sqrt{\mu}}}\right)
$$


Like the heat kernel, the fundamental solution obtained is singular for $x=0, t=0$. Before introducing a way of obtaining computable kernels, it is interesting to look at some properties of $p(t, x)$ :

- The function obtained is a radial basis function both in $x$ and $t$, the $t$ part being based on a 1-norm, while the $x$ part is a standard 2-norm.

- Error function is rapidly converging to 1 when its argument goes to $+\infty$. In fact, $\operatorname{erf}(4)$ is equal to 1 at machine precision when computing with single precision float numbers. It thus means that contributions in the interpolating field coming from aircraft far from the evaluation point are very close to being proportional to a standard $1 /\|x\|$ potential function.

- Unlike classical div-curl splines, commonly used for vector field interpolation [4], [5], these new splines are decreasing at infinity, allowing to drop contributions of far enough aircraft (strictly speaking, this is the case even for the div-curl splines, but decrease is due to cancellations between contributions and is slower).

The main issue in using the kernel $p$ is that the reconstructed field :

$$
X(t, x)=\sum_{i=1}^{N} \lambda_{i} p\left(t-t_{i}, x-x_{i}\right)
$$

is singular at observation points $\left(t_{i}, x_{i}, v_{i}\right)$. A simple way of avoiding this is to change the interpolation criterion : instead of enforcing that $X\left(t_{i}, x_{i}\right)=v_{i}, i=1 \ldots N$, we impose that the mean value of the field in a neighborood of $x_{i}$ has to be equal to $v_{i}$. In order to allow simple computation of the interpolating splines in closed-form, it has been chosen to compute the mean of $X$ with respect to a gaussian density, so that the interpolation condition becomes:

$$
\frac{1}{(2 \pi \sigma)^{3 / 2}} \int_{\mathbb{R}^{3}} e^{-\frac{\left\|x-x_{i}\right\|^{2}}{2 \sigma^{2}}} X(t, x) d x=v_{i}
$$

$\sigma$ is the standard deviation for the gaussian density and is a tuning parameter for the shape of the field. Solving the functional equation with this new interpolation criterion yields a non singular kernel :

$$
p(t, x)=\frac{\sigma}{\|x\|} \operatorname{erf}\left(\frac{\|x\|}{\sigma \sqrt{2+|t|}}\right)
$$

Here again, it worth notice that the asymptotic behaviour of $p$ is to revert to a classical $1 /\|x\|$ potential.

The vector field fitting problem is thus solved with an optimal field of the form :

$$
X(t, x)=\sum_{i=1}^{N} p\left(t-t_{i}, x-x_{i}\right) \lambda_{i}+A x+b
$$

with $\lambda_{i} \in \mathbb{R}^{3}, A$ a $3 \times 3$ matrix and $b \in \mathbb{R}^{3}$ ( $A$ and $b$ account for the term belonging the subspace II.2). The coefficients are found by the interpolation condition :

$$
\forall j=1 \ldots N, \sum_{i=1}^{N} p\left(t_{j}-t_{i}, x_{j}-x_{i}\right) \lambda_{i}+A x_{j}+b=v_{j}
$$

which is a classical linear system. Some care has to be taken in the case of large samples since the matrix involved is dense and may require a large amount of storage : it is much more convenient for samples above 1000 to use an iterative solver (GMRES for example) and to compute the sums on the fly. Multipole methods dedicated to the new splines are under study for that purpose.

The smoothing problem has the same solution, except that the number of terms in the sum is no longer constrained to be the number of measurements. Instead of solving a standard linear system, a least squares formulation is used :

$$
\min \sum_{j=1}^{Q} \sum_{i=1}^{N}\left\|^{2} p\left(\varepsilon_{j}-t_{i}, \xi_{j}-x_{i}\right) \lambda_{i}+A \xi_{j}+b-v_{j}\right\|^{2}
$$

where the measurements are the triples $\left(\varepsilon_{j}, \xi_{j}, v_{j}\right)_{j=1}^{Q}$ and the nodes for the spline smoothing are given by $\left(t_{i}, x_{i}\right)_{i=1}^{N}$. Most of the time, the number of nodes is well below the number of measurements, so that the amount of computation needed is lower than for interpolating. This can be a benefit for analyzing large samples, like those produced when computing complexity on country-sized airspaces.

\section{LYAPUNOV EXPONENTS COMPUTATION}

The metric chosen for complexity computation relies on a measure of sensitivity to initial conditions of the underlying dynamical system called Lyapunov exponents. In order to figure out what Lyapunov exponents are, let consider a point and look at its evolution when transported by the dynamical system.

Let $x$ be fixed (initial point) and let $\gamma$ be a point trajectory of the dynamical system associated to the vector field $X$ given by :

$$
\gamma(t, x)=x+\int_{0}^{t} X(s, \gamma(s, x)) d s
$$

Assume now that trajectory $\gamma$ is disturbed by a small perturbation $\varepsilon$, we have :

$$
\gamma(t, x+\varepsilon)=\gamma(t, x)+\mathbf{D}_{x} \gamma(t, x) \cdot \varepsilon+o(\|\varepsilon\|)
$$

where $\mathbf{D}_{x} \gamma(t, x)$ is the differential of the vector field at $x$ that satisfies :

$$
\frac{\mathbf{D}_{x} \gamma(t, x)}{d t}=\mathbf{D}_{x} X(\gamma(t, x)) \cdot \mathbf{D}_{x} \gamma(t, x)
$$

This equation is a linear differential equation called the cocyle equation. To have simpler notations, we put :

$$
A(t, x)=\mathbf{D}_{x} \gamma(t, x)
$$

We now assume that the trajectory perturbation is such that $\varepsilon(0)=v$. The forward Lyapunov exponent at $x$ and $v$ is defined to be :

$$
\lambda^{+}(x, v)=\limsup _{t \rightarrow+\infty} \frac{1}{t} \log \|\varepsilon(t)\|
$$

It can be shown that only a finite number of different Lyapunov exponents for a given $x$ exist. 
The Lyapunov exponents are closely related to the singular values of the matrix $A(t, x)$ and can be thought as local shear values for the dynamical system.

When Lyapunov exponents are high, the trajectory of a point under the action of the dynamical system is very sensitive to initial conditions (or to the parameters on which the vector field may depend), so that situation in the future is unpredictable. On the other hand, small values of the Lyapunov exponents mean that the future is highly predictable (expected to be comfortable for a controller). So, the Lyapunov exponent map determines the areas where the underlying dynamical system is organized. It identifies the places where the relative distances between aircraft do not change with time (low real value) and the ones where such distance change a lot (hight real value). The practical computation of Lyapunov exponents using the cocyle equation is quite a difficult task :

- By construction $A(t, x)$ tends to grow exponentially fast in some directions (corresponding to positive Lyapunov exponents) and to decay exponentially fast in others (corresponding to negative Lyapunov exponents): the condition number is thus increasing again exponentially.

- A limsup is involved, which is not easy to obtain.

The next Lemma, due to Perron, gives at more tractable characterization of Lyapunov exponents.

Lemma 1: Let :

$$
\frac{d}{d t} v(t)=A(t) v(t)
$$

be a linear differential equation. It exists a smooth mapping $t \rightarrow Q(t)$ with values in the unitary matrices such that :

$$
\frac{d}{d t} Q^{-1}(t) v(t)=T(t) Q^{-1}(t) v(t)
$$

with $T(t)$ an upper triangular matrix.

Because it has some interest for our purpose, we will give an outline of the proof of the lemma. First, take $e_{1}, \ldots e_{n}$ a basis and construct the solutions $e_{1}(t), \ldots e_{n}(t)$ of the differential equation with respective initial conditions $e_{1}, \ldots e_{n}$. Let $E(t)$ be the matrix with columns $e_{1}(t), \ldots e_{n}(t)(E(t)$ describes how the original basis is deformed by the flow). $E(t)$ admits a (smooth) decomposition $E(t)=Q(t) R(t)$ with $Q(t)$ unitary and $R(t)$ upper triangular with positive diagonal elements. It is clear from the definition that :

$$
\frac{d}{d t} E=A E
$$

so that :

$$
A E=\frac{d Q}{d t} R+Q \frac{d R}{d t}
$$

using the fact that $Q$ is unitary and $R$ is invertible :

$$
T={ }^{t} Q A Q-{ }^{t} Q \frac{d Q}{d t}=\frac{d R}{d t} R^{-1}
$$

proving that $T$ is upper triangular as a product of two upper triangular matrices. Now, by the change of variable $y(t)=$
$Q^{-1}(t) v(t)$, we obtain the equation :

$$
\begin{aligned}
\frac{d}{d t} y & =\left({ }^{t} Q A Q-{ }^{t} Q \frac{d Q}{d t}\right) y \\
& =T y
\end{aligned}
$$

which proves the lemma. In the previous representation, the diagonal elements of the matrix $R$ are positive. It is thus possible to write this matrix as :

$$
\left(\begin{array}{cccc}
e^{\mu_{1}} & r_{12} & \ldots & r_{1 n} \\
0 & e^{\mu_{2}} & \ldots & r_{2 n} \\
0 & \ldots & 0 & e^{\mu_{n}}
\end{array}\right)
$$

The matrix $T$ involved in the lemma is equal to $\frac{d R}{d t} R^{-1}$ and can be written as :

$$
\left(\begin{array}{cccc}
\frac{d \mu_{1}}{d t} & \ldots & \ldots & \ldots \\
0 & \frac{d \mu_{2}}{d t} & \ldots & \ldots \\
0 & \ldots & 0 & \frac{d \mu_{n}}{d t}
\end{array}\right)
$$

A very interesting property is that when the Perron lemma is applied to the cocyle matrix $A(t, x)$ with a fixed $x$, the limit :

$$
\lim _{t \rightarrow+\infty} \frac{\mu_{i}}{t}
$$

is precisely a lyapunov exponent at $x$ (and for almost all initial conditions, the sequence of lyapunov exponents obtained by considering diagonal entries of $R$ is in increasing order). Since we are dealing with true limits and not with limsup, this formula gives a procedure for computing Lyapunov exponents.

For practical computations, it is very inefficient to estimate $A(t)=A(t, x)$ and to factor it as $Q(t) R(t)$ time step by time step : a differential equation satisfied by $Q(t)$ and $R(t)$ is solved instead, thus producing in a single step an updated factorization. Based on the equation established before, if $\left.X^{\prime}(\gamma(t))\right)$ is the derivative of the field at time $t$ along the trajectory $\gamma$ :

$$
{ }^{t} Q \frac{Q}{d t}+\frac{d R}{d t} R^{-1}={ }^{t} Q X^{\prime}(\gamma(t)) Q
$$

to simplify the notations, we put :

$$
S(t)={ }^{t} Q X^{\prime}(\gamma(t)) Q
$$

Since ${ }^{t} Q Q=I d:$

$$
\frac{d^{t} Q}{d t} Q=-{ }^{t} Q \frac{Q}{d t}
$$

this matrix is skew-symmetric : its diagonal entries must be 0 . When considering the equation of evolution for these entries only, we obtain that :

$$
\lambda_{i}^{\prime}(t)=S_{i i}(t)
$$

with $\lambda_{i}(t)$ the $i$-th diagonal term of $R$. A simple ordinary differential equation solver can be used to find $\lambda_{i}(t)$ given $S_{i i}(t)$. At the same time, since $\frac{d R}{d t} R^{-1}$ us upper triangular, all the elements of $D={ }^{t} Q \frac{Q}{d t}$ located below the main diagonal can be identified :

$$
\forall i<j, D_{i j}=S_{i j}(t)
$$


Because $D$ is skew-symmetric, all the elements of $D$ are known. Again, a standard differential equation allows to compute $Q(t)$ as the solution of :

$$
\frac{Q}{d t}=Q D
$$

Note that only the diagonal elements of $R$ are useful to compute Lyapunov exponents : the remaining terms can be left unevaluated. The main concern with the algorithm given above is that $Q$ may (and indeed will) fail to be orthogonal during the time evolution. Since this property is required, any algorithm computing Lyapunov exponents has to correct $Q$ from time to time. An interesting alternative approach has been presented in [6] : instead of periodically orthogonalizing $Q$, a representation is chosen so that orthogonality of $Q$ is guaranteed. It is well known that any rotation matrix in dimension $n$ can be obtained as a product of $n(n-1) / 2$ elementary Givens' rotations with angles $\theta_{i j}, i=1 \ldots n, i<j$. The parametrization of $Q$ is obtained precisely by the $\theta_{i j}$. The details can be found in the original article. It has to been noted however that the method is interesting mainly in low dimension, which is our case $(n=3)$. This algorithm has been successfully implemented in our application. The main drawback of this implementation is that a lot of trigonometric computations have to be done, but matrices involved are only $3 \times 3$.

\section{RESULTS}

We give below two examples of complexity maps. In the first example, a synthetic traffic has been generated with a well defined structure :

- Two areas with dense traffic and quasi-random positions and velocities (airspeed is nearly constant as in real situations, but heading is randomly sampled). It is expected that in this areas many conflicts occur with interdepedance, that is solving a conflict will induce other conflicts.

- A path with dense traffic,but well organized (it is a common organization in USA called miles-in-trail).

The resulting complexity map appears in figure 1 . The picture clearly shows that even if the traffic is dense on the miles-in-trail path, the complexity remains low because of the high structuration. On the opposite, many hot spots appear in the two quasi-random areas.

The second example (figure 2) is real traffic over France, at $8 \mathrm{am}$. While the resulting complexity map is more difficult to analyze because of the size of the airspace, it can be noted that major airports are considered as high complexity areas. As before, low complexity occurs in low density or highly structured traffic.

Computation time is for less than one hundred aircraft and 10 past or future positions in the order of 10 seconds on a dual XEON 5400 workstation. It is possible to adress country-size airspaces within less than one minute on a cluster of 4 workstation using GPU computing.

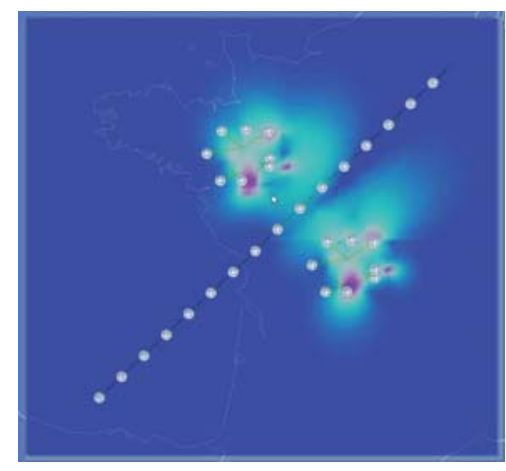

Fig. 1. Low complexity valley

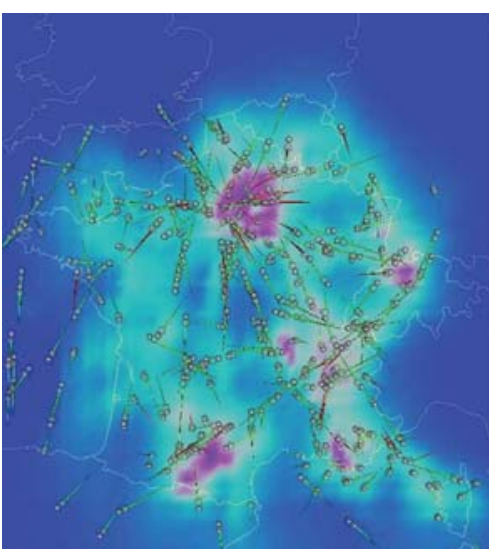

Fig. 2. Traffic over France

\section{CONCLUSION}

The approach based on modelling the air traffic by a dynamical system and to assess complexity values to points in airspace by computating the associated Lyapunov exponents appears to be a powerful tool for future trajectory based ATM systems. Most of the work has been devoted to improvements in interpolating splines used to fit a vector field to the observations and to efficient and stable computation of Lyapunov exponents. Future directions of research will focus on computational speed enhancement, with countrysized airspaces in mind. Furthermore, a trajectory planner generating conflict-free trajectories that are optimal with respect to complexity is currently investigated in the context of a $\mathrm{PhD}$ thesis.

\section{REFERENCES}

[1] Eurocontrol, ATFCM and Capacity Report 2006. Eurocontrol, 2006.

[2] B. Hilburn, "Cognitive complexity in air traffic control : A literature review," EEC, Tech. Rep. 04/04, 2004.

[3] R. Mogford, J. Guttman, and S. M. P. Kopardekar, "The complexity construct in air traffic control : A review and synthesis of the literature," FAA, Tech. Rep. DOT/FAA/CT-95/22, 1995.

[4] D. Suter, "Motion estimation and vector splines."

[5] F. Chen and D. Suter, "div - curl vector quasi-interpolation on a finite domain," Monash University, Clayton Campus, Vic. 3168, Tech. Rep., 1997.

[6] T. Janaki, G. Rangarajan, S. Habib, and D. Ryne, "Computation of the lyapunov spectrum for continuous-timpe dynamical systems and discrete maps," Physical Review, vol. 60, no. 6, 1999. 